\title{
EXISTENCE AND MULTIPLICITY OF SOLUTIONS TO DISCRETE INCLUSIONS WITH THE $p(k)$-LAPLACIAN PROBLEM OF KIRCHHOFF TYPE
}

\author{
ABDOUL AZIZ KALIFA DIANDA ${ }^{1}$, STANISLAS OUARO ${ }^{2, *}$ \\ ${ }^{1}$ Department of Mathematics, UFR. Sciences et Technologies, Université Norbert Zongo, Burkina Faso \\ ${ }^{2}$ Department of Mathematics, UFR. Sciences Exactes et Appliquées,Université Joseph KI-ZERBO, 03 BP 7021 Ouaga 03, Burkina Faso \\ * Corresponding author: ouaro@yahoo.fr
}

Received Feb. 10, 2020

\begin{abstract}
AвSTRACT. In this paper, we prove the existence and multiplicity of solutions to generalized discrete inclusions with the $p(k)$-Laplacian problem of Kirchhoff type subjected to potential boundary values conditions. We focus on the existence of three solutions with the help of linking arguments and new non-smooth three critical points theorem.
\end{abstract} 2010 Mathematics Subject Classification. 47A75, 35B38, 35P30, 34L05, 34L30.

Key words and phrases. discrete periodic boundary value; multiple solution; $p(k)$-Laplacian; discrete inclusion; three critical points theorem.

\section{INTRODUCTION}

This article deals with the existence and multiplicity of solutions to generalized discrete inclusions problems of the form

$$
\left\{\begin{array}{l}
-M(A(k-1, \Delta u(k-1))) \Delta(a(k-1, \Delta u(k-1))) \in \lambda \partial F(k, u(k)), \quad k \in \mathbb{Z}[1, T] \\
(a(0, \Delta u(0)),-a(T, \Delta u(T)) \in \partial j(u(0) ; u(T+1))
\end{array}\right.
$$

where, $\lambda$ is a positive real parameter; $T>2$ is a positive integer and $\Delta u(k)=u(k+1)-u(k)$ is the forward difference operator. Here and hereafter, we denote by $\mathbb{Z}[a, b]$ the discrete interval $\{a, a+1, a+2, \ldots b\}$, where $a$ and $b$ are integers with $a<b . u(k) \in \mathbb{R}$ for all $k \in \mathbb{Z} ; j: \mathbb{R} \times \mathbb{R} \rightarrow(-\infty,+\infty]$ is convex, proper (i.e. $D(j):=\{z \in \mathbb{R} \times \mathbb{R}: j(z)<+\infty\} \neq \emptyset$ ), lower semicontinuous (in short l.s.c) and $\partial j$ denote the subdifferential of $j$, where, for $z \in \mathbb{R} \times \mathbb{R}$, the set $\partial j$ is defined by

$$
\partial j(z)=\{\zeta \in \mathbb{R} \times \mathbb{R}: j(\xi)-j(z) \geq(\zeta \mid \xi-z), \text { for all } \xi \in \mathbb{R} \times \mathbb{R}\}
$$

DOI: 10.28924/APJM/7-6 
where $(. \mid$.$) stands for the usual inner product in \mathbb{R} \times \mathbb{R}$.

$F: \mathbb{Z} \times \mathbb{R} \longrightarrow \mathbb{R}$ is a nonlinear function and satisfy:

$\left(a_{1}\right) F$ is periodic with respect to $k$, i.e. there exists $m \in \mathbb{Z}$ such that $F(k+m, u)=F(k, u)$ for all $(k, u) \in \mathbb{Z} \times \mathbb{R}$;

$\left(a_{2}\right) F(k,$.$) is locally Lipschitz continuous for all k \in \mathbb{Z}$;

$\left(a_{3}\right) F(k, 0)=0$ for all $k \in \mathbb{Z}$.

$\partial F(k, u)$ is the Clarke subdifferential of the function $F$ with respect to the second variable.

We also consider the fonction space $X=\{v:\{0,1, \ldots T+1\} \rightarrow \mathbb{R}\}$ with the inner product

$$
(u, v)=\sum_{k=1}^{T+1} \Delta u(k-1) \Delta v(k-1), \text { for all } u, v \in X .
$$

$M:(0,+\infty) \rightarrow(0,+\infty)$ is continuous and nondecreasing and there exist positive reals $B_{1}, B_{2}$ with $B_{1} \leq B_{2}$ and $\alpha \geq 1$ such that

$$
B_{1} t^{\alpha-1} \leq M(t) \leq B_{2} t^{\alpha-1} \quad \text { for } \quad t \geq t^{*}>0 .
$$

In general the equations posed in the boundary value problem model different phenomena in areas such as economy, biology, physics, mechanics, computer science and finance. In recent years, several variant of the boundary value problem have been widely studied by many authors using several techniques. See the references $[1-6,11-14,16,17,20,22,23]$.

While there are few papers dealing discrete inclusions with the laplacian problem, involving problem of this type, the use of various critical point methods gives rise to other difficulties which makes the problem interesting. For instance, in [15] the authors dealed the following problem.

$$
\sum_{i=1}^{T} a_{k l} u_{l} \in\left[g_{k}^{-}\left(u_{k}\right), g_{k}^{+}\left(u_{k}\right)\right], \quad \forall k \in \mathbb{Z}[1, T],
$$

where $A=\left(a_{i j}\right)_{T \times T}$ is a symetric positive definite matrix and $g_{k}^{-}(t)=\lim _{\delta \rightarrow 0^{+}}$ess $\inf _{|\xi-t|<\delta} g_{k}(\xi), g_{k}^{+}(t)=$ $\lim _{\delta \rightarrow 0^{+}}$ess $\sup _{|\xi-t|<\delta} g_{k}(\xi)$. They used a non-smooth abstract result to prove the existence of at least one nontrivial solution of an alegebraic discrete inclusion. Successively, they established a multiplicity result for the same class of discrete problem using a locally lipschitz continuous version of the famous Brezis-Nirenberg theorical result in presence of spliting. Another example is the following problem, see [9].

$$
\left\{\begin{array}{l}
-\Delta\left(|\Delta u(k-1)|^{p(k-1)-2} \Delta u(k-1)\right) \in \lambda \partial F(k, u(k)), \\
u(k+m)=u(k), k \in \mathbb{Z},
\end{array}\right.
$$

where the focus was on the existence of three solutions with the aid of linking arguments and new non-smooth three critical points tool.

Problem (1.1) can be viewed as a generalization of problem (1.5). In our paper, we prove the existence and multiplicity of solutions to the problem (1.1) by the critical point methods.

The plan of our work is as follows. In section 2, we presente some basic and useful definitions and notations for 
this work. In sections 3 and 4, we recall the new abstract critical points theorems established in [9] and we prove the multiplicity of solutions to problem (1.1).

\section{BASIC DEFINITIONS AND PRELIMINARY RESULTS}

Let $(E,\|\|$.$) be a real Banach space. We denote by E^{*}$ the dual space of $E$, while $\langle$,$\rangle stands for the duality pairing$ between $E^{*}$ and $E$.

A function $J: E \longrightarrow \mathbb{R}$ is called locally Lipschitz continuous, if for every $u \in E$, there exist a neighbourhood $V_{u}$ of $u$ and a constant $L_{u} \geq 0$ such that

$$
|J(z)-J(w)| \leq L_{u}\|z-w\| \text { for all } z, w \in V_{u} .
$$

If $u, z \in E$, we write $J^{0}(u ; z)$ for the generalized directional derivative of $J$ at the point $u$ along the direction $z$, i.e.

$$
J^{0}(u ; z):=\lim _{w \rightarrow u} \sup _{t \rightarrow 0^{+}} \frac{J(w+t z)-J(w)}{t} .
$$

The generalized gradient of the function $J$ in $u$, denoted by $\partial J(u)$, is the set

$$
\partial J(u):=\left\{u^{*} \in E^{*}:\left\langle u^{*}, z\right\rangle \leq J^{0}(u ; z) \text {, for all } z \in E\right\} .
$$

The basic properties of generalized directional derivative and generalized gradient were studied in $[7,8]$.

We recall that if $J$ is continuously Gâteaux differentiable at $u$, then $J$ is locally Lipschitz at $u$ and $\partial J(u)=\left\{J^{\prime}(u)\right\}$, where $J^{\prime}(u)$ stands for the first derivative of $J$ at $u$.

Furthermore, a point $u$ is called a (generalized) critical point of the locally Lipschitz continuous function $J$, if $0_{E^{*}} \in \partial J(u)$, i.e.

$$
J^{0}(u ; z) \geq 0, \text { for every } z \in E \text {. }
$$

Clearly, if $J$ is continuously Gateaux differentiable at $u$, then $u$ becomes a (classical) critical point of $J$, that is $J^{\prime}(u)=0_{E^{*}}$.

A locally Lipschitz continuous functional $J: E \longrightarrow \mathbb{R}$ is said to fulfil the Palais-Smale (PS) condition if every sequence $\left\{u_{n}\right\}$ in $E$ such that $\left\{J\left(u_{n}\right)\right\}$ is bounded and

$$
J^{0}\left(u_{n} ; u-u_{n}\right) \geq-\epsilon_{n}\left\|u-u_{n}\right\|
$$

for all $u \in E$, where $\epsilon_{n} \longrightarrow 0^{+}$as $n \longrightarrow \infty$, possesses a convergent subsequence.

Definition 2.1. A function $f$ defined on a normed space $X$ to $\overline{\mathbb{R}}$ is said to be coercive over an unbounded part $P$ of $X$ if $\lim _{\|x\| \longrightarrow+\infty} f(x)=+\infty$, where $x \in P . f$ is said to be anti-coercive if $(-f)$ is coercive.

For a complete overview on the non-smooth calculus we refer the readers to the paper [18]. Furthermore, let's cite a recent book [14] as a general reference on the subject of our paper.

Our main tool will be the following abstract critical points theorems, for locally Lipschitz continuous functions that we recall here, for completeness, in their general form. 
Theorem 2.2. ( [21], Theorem 2.3) Let $E$ be a real Banach space. Assume that $E:=E_{1} \oplus E_{2}$, with $E_{2}$ a finite-dimensional subspace of $E$. Let $J: E \longrightarrow \mathbb{R}$ be a locally Lipschitz continuous functional satisfying the (PS) condition and such that

$J(u) \leq 0,\left(\forall u \in \bar{B}(0, \rho) \cap E_{2}\right)$,

$J(u) \geq 0,\left(\forall u \in \bar{B}(0, \rho) \cap E_{1}\right)$,

for some $\rho>0$.

Assume also that $J$ is bounded from below and $\inf _{u \in E} J(u)<0$. Then, $J$ has at least two non-zero critical points.

Theorem 2.3. [19] Let $(E,\|\|$.$) be a real Banach space and let J: E \longrightarrow \mathbb{R}$ be a locally Lipschitz continuous functional satisfying (PS) condition. If there exist $u_{1}, u_{2} \in E, u_{1} \neq u_{2}$ and $r \in\left(0,\left\|u_{2}-u_{1}\right\|\right)$ such that $\inf \left\{J(u):\left\|u-u_{1}\right\|=r\right\} \geq \max \left\{J\left(u_{1}\right), J\left(u_{2}\right)\right\}$, then

$c:=\inf _{\gamma \in \Gamma} \max _{s \in[0,1]} J(\gamma(s)) \geq \max \left\{J\left(u_{1}\right), J\left(u_{2}\right)\right\}$ is a critical value for $E$ and $K_{c}-\left\{u_{1}, u_{2}\right\} \neq \emptyset$, where $K_{c}$ is the set of critical points at the level $c$ and $\Gamma$ the family of continuous paths $\gamma:[0,1] \longrightarrow E$ joining $u_{1}$ and $u_{2}$.

Now, we make the following assumptions that we will use throughout our work.

$$
A: \mathbb{Z}[1, T] \times \mathbb{R} \rightarrow \mathbb{R}, \text { with } A(k, 0)=0 \text { and } a(k, \xi)=\frac{\partial}{\partial \xi} A(k, \xi), \text { for all } k \in \mathbb{Z}[0, T]
$$

$$
\text { There exists a constant } C_{1}>0 \text { such that }|a(k, \xi)| \leq C_{1}\left(1+|\xi|^{p(k)-1}\right) \text {, for all } k \in \mathbb{Z}[0, T] \text {. }
$$

$$
(a(k, \xi)-a(k, \eta)) \cdot(\xi-\eta)>0, \text { for all } k \in \mathbb{Z}[0, T] \text { and } \xi, \eta \in \mathbb{R} \text { such that } \xi \neq \eta \text {. }
$$

$p$ is a $T$-periodic function i.e. $p(k+T)=p(k)$, for all $k \in \mathbb{Z}[0, T]$.

$$
|\xi|^{p(k)} \leq a(k, \xi) \xi \leq p(k) A(k, \xi), \xi \in \mathbb{R} \text { and } p: \mathbb{Z} \longrightarrow[2, \infty) \text { for all } k \in \mathbb{Z}[0, T]
$$

\footnotetext{
$a$ is a $T$-periodic function with respect to $k$ i.e. $a(k+T, \xi)=a(k, \xi)$ for all $(k, \xi) \in \mathbb{Z}[0, T] \times \mathbb{R}$.
}

\section{THREE CRITICAL POINTS THEOREM FOR LOCALLY LIPSCHITZ FUNCTIONALS}

In this section we recall the tools used in [9]. 
Theorem 3.1. Let $(X, \tau)$ be an Hausdorff space and $\Phi, J: X \longrightarrow \mathbb{R}$ be functionals. Let $M$ be the set (possibly empty) of all global minimizers of $J$ and define

$$
\begin{gathered}
\alpha:=\inf _{x \in X} \Phi(x), \\
\beta:= \begin{cases}\inf _{x \in M} \Phi(x) & \text { if } M \neq \emptyset \\
\sup _{x \in X} \Phi(x) & \text { if } M=\emptyset .\end{cases}
\end{gathered}
$$

Let $\alpha<\beta$ and assume that the set

$$
\{x \in X: \Phi(x)+\sigma J(x) \leq \rho\},
$$

for every $\sigma>0$ and every $\rho \in \mathbb{R}$, is sequentially compact (if not empty). Then at least one of the following conditions holds.

(a) There exists a continuous mapping $h:(\alpha, \beta) \longrightarrow X$ with the following property: for every $t \in(\alpha, \beta)$,

$$
\Phi(h(t))=t
$$

and for every $x \in \Phi^{-1}(t)$ with $x \neq h(t)$,

$$
J(x)>J(h(t)) .
$$

(b) There exists $\lambda>0$ such that the functional $\Phi+\lambda J$ admits at least two global minimizers in $X$.

Theorem 3.2. Let $E$ be a finite dimensional real Banach space. Let $\mu: E \longrightarrow \mathbb{R}$ be a coercive $C^{1}$ functional such that $\mu(0)=0$ and let $J: E \longrightarrow \mathbb{R}$ be locally Lipschitz. Let $s>0$ and $0<r<s$ be fixed. Assume that

$\left(b_{1}\right) \lim _{\mu(u) \longrightarrow+\infty} \inf \frac{J(u)}{\mu(u)} \geq 0 ;$

$\left(b_{2}\right) \inf _{u \in E} J(u)<\inf _{\mu(u) \leq s} J(u)$;

$\left(b_{3}\right) J(0) \leq \inf _{r \leq \mu(u) \leq s} J(u)$.

Then, there exists $\lambda>0$ such that the functional $\mu+\lambda J$ has at least three critical points in $E$, at least two of which are non-trivial.

Theorem 3.3. Let $E$ be a finite dimensional real Banach space. Let $\mu: E \longrightarrow \mathbb{R}$ be a coercive $C^{1}$ functional such that $\mu(0)=0$ and let $J: E \longrightarrow \mathbb{R}$ be a locally lipschitz functional bounded from below. Let $s>0$ and $0<r<s$ be fixed constants. Assume moreover that conditions $\left(b_{2}\right)$ and $\left(b_{3}\right)$ hold. Then there exists $\lambda>0$ such that the functional $\mu+\lambda J$ has at least three critical points in $E$. 


\section{VARIATIONAL FRAMEWORK AND AUXILIARY RESULTS}

Now, we recall the following notations that we will use throughout the paper.

$$
p^{+}:=\max _{k \in \mathbb{Z}[1, T]} p(k) \text { and } p^{-}:=\min _{k \in \mathbb{Z}[1, T]} p(k),
$$

where $\mathbb{Z}[1, T]:=\{1,2, \ldots T\}$.

Define the space

$$
H_{T}=\left\{u=\{u(k)\}_{k \in \mathbb{Z}}: u(k) \in \mathbb{R}, u(k+T)=u(k), k \in \mathbb{Z}\right\},
$$

which equipped with the Euclidean norm,

$$
\|u\|_{e}:=\left(\sum_{k=1}^{T}|u(k)|^{2}\right)^{\frac{1}{2}}
$$

is an Hilbert space.

Put for any $a \in \mathbb{R}$,

$W_{a}:=\left\{u=\{u(k)\}_{k \in \mathbb{Z}}: u(k)=a, k \in \mathbb{Z}\right\}$ and $Y_{a}:=W_{a}^{\perp}$.

Thus, $W_{a}$ consists of constant sequences and we have an orthogonal decomposition

$$
H_{T}=Y_{a} \oplus W_{a}, \text { for any } a \in \mathbb{R} \text {. }
$$

Using the functional $j$, we introduce the functional $J: H_{T} \longrightarrow(-\infty ; \infty)$ given by

$$
J(u)=j(u(0) ; u(T+1)), \quad \forall u \in H_{T}
$$

Note that, as $j$ is proper, convex and l.s.c, the same properties hold true for $J$.

The energy functional corresponding to (1.1) is

$$
\Gamma_{T}(u)=\widehat{M}\left(\sum_{k=1}^{T+1} A(k-1, \Delta u(k-1))\right)-\lambda \sum_{k=1}^{T} F(k, u(k))+J(u),
$$

where $\widehat{M}(t)=\int_{0}^{t} M(s) d s$.

Definition 4.1. A solution of problem (1.1) is a function $u \in H_{T}$ such that

$$
\begin{aligned}
-\sum_{k=1}^{T} F^{0}(k ; u(k)) w(k) & -M\left(\sum_{k=1}^{T+1} A(k-1, \Delta u(k-1))\right) \sum_{k=1}^{T+1} a(k-1, \Delta u(k-1)) \Delta h(k-1) \\
& +j^{\prime}((u(0), u(T+1)),(h(0), h(T+1))) \geqslant 0
\end{aligned}
$$

for all $h \in H_{T}$ with $h(k) \geq 0$ and for all $k \in \mathbb{Z}$.

Proposition 4.2. Assume that (1.3), (2.1), (2.2) and $\left(a_{1}\right)$ hold. Then, $\Gamma_{T}$ is locally Lipschitz continuous. 
Proof.

$$
\begin{aligned}
|\Gamma(z)-\Gamma(w)| & =\widehat{M}\left(\sum_{k=1}^{T+1} A(k-1, \Delta z(k-1))\right)-\widehat{M}\left(\sum_{k=1}^{T+1} A(k-1, \Delta w(k-1))\right) \\
& -\lambda \sum_{k=1}^{T} F(k, z(k))+\lambda \sum_{k=1}^{T} F(k, w(k))+J(z)-J(w) \mid \\
\leq & \widehat{M}\left(\sum_{k=1}^{T+1} A(k-1, \Delta z(k-1))\right)-\widehat{M}\left(\sum_{k=1}^{T+1} A(k-1, \Delta w(k-1))\right) \mid \\
& +\lambda \sum_{k=1}^{T}|F(k, z(k))-F(k, w(k))|+|J(z)-J(w)| \\
& \mid \int_{k=1}^{T+1} A(k-1, \Delta w(k-1)) \\
& \sum_{k=1}^{T+1} M(t) d t\left|+\lambda \sum_{k=1}^{T}\right| F(k, z(k))-F(k, w(k))|+| J(z)-J(w) \mid \\
& \leq \sum_{k=1}^{T+1} A(k-1, \Delta z(k-1)) \\
& \sum_{k=1}^{T+1} A(k-1, \Delta w(k-1)) \\
\leq & \frac{B_{2}}{\alpha}\left[\left(\sum_{k=1}^{T+1} A(k-1, \Delta z(k-1))\right)^{\alpha}-\left(\sum_{k=1}^{T+1} A(k-1, \Delta w(k-1))\right)^{\alpha}\right] \\
+ & \lambda \sum_{k=1}^{T}|F(k, z(k))-F(k, w(k))|+|J(z)-J(w)| . \\
& =\sum_{k=1}^{T}|F(k, z(k))-F(k, w(k))|+|J(z)-J(w)| \\
&
\end{aligned}
$$

If $w=z$ then, $J(z)=J(w)$. Consequently, $\exists K_{1}>0$ such that $|\Gamma(z)-\Gamma(w)| \leq K_{1}\|z-w\|_{e}$.

If $z \neq w$ then, $\|w-z\| \neq 0$ and since the quantity $\left[\left(\sum_{k=1}^{T+1} A(k-1, \Delta z(k-1))\right)^{\alpha}-\left(\sum_{k=1}^{T+1} A(k-1, \Delta w(k-1))\right)^{\alpha}\right]$ is finite then, $\exists K_{2}>0$ such that

$$
\left[\left(\sum_{k=1}^{T+1} A(k-1, \Delta z(k-1))\right)^{\alpha}-\left(\sum_{k=1}^{T+1} A(k-1, \Delta w(k-1))\right)^{\alpha}\right] \leq K_{2}\|z-w\|_{e} .
$$

Consequently, since $F(k,$.$) is locally Lipschitz continuous then for all u \in H_{T}$, there exist a neighbourhood $V_{u}$ of $u$ and a constant $C_{2} \geq 0$ such that $|F(k, z(k))-F(k, w(k))| \leq C_{2}\|z-w\|_{e}$. As $J$ is lower semi-continuous, proper, then $J$ is also locally Lipschitz continuous. Therefore, for all $u \in H_{T}$, there exist a neighbourhood $V_{u}^{\prime}$ of $u$ and a constant $C_{2}^{\prime} \geq 0$ such that $|J(z)-J(w)| \leq C_{2}^{\prime}\|z-w\|_{e}$.

It follow that

$$
\begin{aligned}
|\Gamma(z)-\Gamma(w)| & \leq \frac{B_{2}}{\alpha} K_{2}\|z-w\|_{e}+\lambda T C_{2}\|z-w\|_{e}+C_{2}^{\prime}\|z-w\|_{e} \\
& \leq L\|z-w\|_{e}, \text { with } L=\frac{B_{2}}{\alpha} K_{2}+\lambda T C_{2}+C_{2}^{\prime} .
\end{aligned}
$$

Lemma 4.3. Let $u \in H_{T}$ be a critical point of $\Gamma_{T}$. Then, u satisfies problem (1.1).

Proof. 
Assume that $u \in H_{T}$ is a critical point of $\Gamma_{T}$. Then, for any $h \in H_{T}, \Gamma^{0}(u ; h) \geq 0$.

We have

$$
\begin{aligned}
\Gamma(w+t h) & =\widehat{M}\left(\sum_{k=1}^{T+1} A(k-1, \Delta(w+t h)(k-1))\right)-\lambda \sum_{k=1}^{T} F(k,(w+t h)(k))+J(w+t h) \\
& =\widehat{M}\left(\sum_{k=1}^{T+1} A(k-1, \Delta w(k-1)+t \Delta h(k-1))\right)-\lambda \sum_{k=1}^{T} F(k, w(k)+t h(k))+J(w+t h) ;
\end{aligned}
$$

consequently

$$
\begin{aligned}
\Gamma(w+t h)-\Gamma(w) & =\widehat{M}\left(\sum_{k=1}^{T+1} A(k-1, \Delta w(k-1)+t \Delta h(k-1))\right)-\widehat{M}\left(\sum_{k=1}^{T+1} A(k-1, \Delta w(k-1))\right) \\
& -\lambda \sum_{k=1}^{T} F(k, w(k)+t h(k))+\lambda \sum_{k=1}^{T} F(k, w(k))+J(w+t h)-J(w) \\
& =\widehat{M}\left(\sum_{k=1}^{T+1} A(k-1, \Delta w(k-1)+t \Delta h(k-1))\right)-\widehat{M}\left(\sum_{k=1}^{T+1} A(k-1, \Delta w(k-1))\right) \\
& -\lambda\left[\sum_{k=1}^{T} F(k, w(k)+t h(k))-\sum_{k=1}^{T} F(k, w(k))\right]+J(w+t h)-J(w) .
\end{aligned}
$$

By dividing above equality by $t$ and by making $t$ tend towards 0 and $w$ towards $u$, we obtain

$$
\Gamma^{0}(u ; h)=M\left(\sum_{k=1}^{T+1} A(k-1, \Delta u(k-1))\right) \sum_{k=1}^{T+1} a(k-1, \Delta u(k-1)) \Delta h(k-1)-\lambda \sum_{k=1}^{T} F^{0}(k ; u(k)) h(k)+j^{\prime}(u ; h) .
$$

Using Abel's summation by parts formula, we get

$$
\begin{aligned}
\sum_{k=1}^{T+1} a(k-1, \Delta u(k-1)) \Delta h(k-1) & =\sum_{k=1}^{T+1} a(k-1, \Delta u(k-1)) h(k)-\sum_{k=1}^{T+1} a(k-1, \Delta u(k-1)) h(k-1) \\
& =\sum_{k=1}^{T+1} a(k-1, \Delta u(k-1)) h(k)-\sum_{k=0}^{T} a(k, \Delta u(k)) h(k) \\
& =\sum_{k=1}^{T} a(k-1, \Delta u(k-1)) h(k)-\sum_{k=1}^{T} a(k, \Delta u(k)) h(k) \\
& -a(0, \Delta u(0)) h(0)+a(T, \Delta u(T)) h(T+1) .
\end{aligned}
$$

It follows that

$$
\sum_{k=1}^{T+1} a(k-1, \Delta u(k-1)) \Delta h(k-1)=-\sum_{k=1}^{T} \Delta(a(k-1, \Delta u(k-1))) h(k)-a(0, \Delta u(0)) h(0)+a(T, \Delta u(T)) h(T+1) .
$$

As $h \in H_{T}$, is arbitrary chosen, thus if $h(0)=h(T+1)=0$ and $\Gamma^{0}(u ; h) \geq 0$ we obtain $M\left(\sum_{k=1}^{T+1} A(k-1, \Delta u(k-1))\right) \sum_{k=1}^{T+1}-\Delta\left(a(k-1, \Delta u(k-1)) h(k)-\lambda \sum_{k=1}^{T} F^{0}(k ; u(k)) h(k) \geq 0\right.$, for all $k \in \mathbb{Z}[1, T]$.

This means that $0_{H_{T}^{*}} \in \partial \Gamma(u)$ and thus $u$ satisfies $(1.1)$. 
It remains to show that $(a(0, \Delta u(0)),-a(T, \Delta u(T))) \in \partial j(u(0), u(T+1))$. One has

$$
\begin{array}{r}
-\sum_{k=1}^{T} F^{0}(k ; u(k)) h(k)-M\left(\sum_{k=1}^{T+1} A(k-1, \Delta u(k-1))\right) \sum_{k=1}^{T} \Delta a(k-1, \Delta u(k-1)) h(k)+ \\
M\left(\sum_{k=1}^{T+1} A(k-1, \Delta u(k-1))\right)[a(T, \Delta u(T)) h(T+1)-a(0, \Delta u(0)) h(0)]+ \\
j^{\prime}((u(0), u(T+1)),(h(0), h(T+1))) \geqslant 0
\end{array}
$$

and

$$
M\left(\sum_{k=1}^{T+1} A(k-1, \Delta u(k-1))\right) \sum_{k=1}^{T}\left(-\Delta a(k-1, \Delta u(k-1)) h(k)-\sum_{k=1}^{T} F^{0}(k, u(k)) h(k) \geq 0 .\right.
$$

Let

$$
C=\left\{\begin{array}{ccc}
B_{1} & \text { if } & -a(T, \Delta u(T))+a(0, \Delta u(0)) \geq 0 \\
& & \\
B_{2} & \text { if } & -a(T, \Delta u(T))+a(0, \Delta u(0)) \leq 0 .
\end{array}\right.
$$

As $M($.$) is positive and from (1.3), it follows that$

$$
\begin{aligned}
& j^{\prime}((u(0), u(T+1)),(h(0), h(T+1))) \geqslant C\left(\sum_{k=1}^{T+1} A(k-1, \Delta u(k-1))\right)^{\alpha-1} \times \\
& {[-a(T, \Delta u(T)) h(T+1)+a(0, \Delta u(0)) h(0)] . }
\end{aligned}
$$

Now we take $s=C\left(\sum_{k=1}^{T+1} A(k-1, \Delta u(k-1))\right)^{\alpha-1}$.

Thus,

$$
j^{\prime}((u(0), u(T+1)),(h(0), h(T+1))) \geqslant-a(T, \Delta u(T))(\operatorname{sh}(T+1))+a(0, \Delta u(0))(\operatorname{sh}(0)) .
$$

Finally, for all $h \in H_{T}$, taking $\operatorname{sh}(0)=p$ and $s h(T+1)=q$, where $p, q \in \mathbb{R}$ are arbitrarily chosen, it follows that

$$
j^{\prime}((u(0), u(T+1)),(h(0), h(T+1))) \geqslant-a(T, \Delta u(T)) q+a(0, \Delta u(0)) p, \quad \text { for } \quad p, q \in \mathbb{R} .
$$

Hence, $(a(0, \Delta u(0)),-a(T, \Delta u(T))) \in \partial j(u(0), u(T+1))$

Now, we recall some auxiliary results, which we use later on, see [8].

Lemma 4.4. The following properties hold.

$\left(d_{1}\right)$ For every $s>0$,

$$
\sum_{k=1}^{T}|u(k)|^{s} \leqslant T\|u\|_{e}^{s}, \text { for all } u \in H_{T}
$$

$\left(d_{2}\right)$ For every $s \geqslant 2$,

$$
\sum_{k=1}^{T}|u(k)|^{s} \geqslant T^{\frac{(2-s)}{2}}\|u\|_{e}^{s}, \text { for all } u \in H_{T}
$$

$\left(d_{3}\right)$ For all $u \in H_{T}$,

$$
\sum_{k=1}^{T}|\triangle u(k-1)|^{p(k-1)} \leqslant T\left(2^{p^{+}}\|u\|_{e}^{p^{+}}+1\right)
$$




\section{Multiple solutions of Problem (1.1) By APplying TheOREM 2.2}

In this section, we prove the existence of $T$-periodic solutions by appliying Theorem 2.2. Assume that $F$ satisfies additionally the following.

$\left(d_{4}\right)$ There exist $T$-periodic functions $s: \mathbb{Z} \longrightarrow[2 ; \infty), \alpha_{1}: \mathbb{Z} \longrightarrow(0, \infty)$ and a function $\alpha_{2}: \mathbb{Z} \longrightarrow \mathbb{R}$ for which

$$
F(k, u) \geq \alpha_{1}(k)|u|^{s(k)}+\alpha_{2}(k),
$$

for all $k \in \mathbb{Z}$ and $u \in \mathbb{R}^{n}$ such that $|u| \geq S$, where $S \geq 1$ is fixed and sufficiently large.

Put $s^{-}:=\min _{k \in \mathbb{Z}[1, T]} s(k) ; \quad \alpha_{1}^{-}:=\min _{k \in \mathbb{Z}[1, T]} \alpha_{1}(k) ; \alpha_{2}^{-}:=\min _{k \in \mathbb{Z}[1, T]} \alpha_{2}(k)$, where $s, \alpha_{1}, \alpha_{2}$ are functions defined above.

Assume further that

$\left(d_{5}\right) \lim _{|u| \rightarrow 0} \frac{F(k, u)}{|u|^{s^{-}}}=0$ uniformly in $k \in \mathbb{Z}$.

$\left(d_{6}\right)$ There exist constants $\omega_{0}, \omega_{1}, \omega_{2}>0$, with $\omega_{2}>\omega_{1}>\omega_{0}$, such that for all $k \in \mathbb{Z}$ :

$\left(d_{6}^{\prime}\right) F(k, u) \geq 0$ with $|u| \leq \omega_{0}$;

$\left(d_{6}^{\prime \prime}\right) F(k, u)<0$ with $\omega_{1} \leq|u| \leq \omega_{2}$.

Example 5.1. As examples of functions satisfying assumptions $\left(a_{1}\right)-\left(a_{3}\right)$ and $\left(d_{4}\right)-\left(d_{6}\right)$ we can give the following, where $T \geq 2$ is a fixed even natural number.

(1) $F: \mathbb{Z} \times \mathbb{R} \longrightarrow \mathbb{R}$ given by

$$
F(k, t):=\left\{\begin{array}{l}
16 t^{4} \text { if }|t| \leq \frac{1}{2} \\
-4|t|+3 \text { if }|t| \in\left(\frac{1}{2}, 1\right) \\
t^{4}+|t-1|-2 \text { if }|t| \geq 1
\end{array}\right.
$$

and

(2) $s: \mathbb{Z} 1, T] \rightarrow[2, \infty)$ such that

$$
s(k):=\left\{\begin{array}{l}
4 \text { if } k=2 l, \\
2 \text { if } k=2 l-1 ; l \in\left[1, \frac{T}{2}\right] .
\end{array}\right.
$$

Lemma 5.1. Assume that conditions (2.1), (2.2), (1.3) and $\left(d_{4}\right)$ hold with $s^{-}>\alpha p^{+}$. Then, the functional $\Gamma_{T}$ is anti-coercive on $H_{T}$, for all $\lambda>0$.

Proof.

$\Gamma_{T}(u)=\widehat{M}\left(\sum_{k=1}^{T+1} A(k-1, \Delta u(k-1))\right)-\lambda \sum_{k=1}^{T+1} F(k, u(k))+J(u(0), u(T+1))$.

One can use (2.1) to say that

$$
A(k, \xi)=\int_{0}^{\xi} a(k, \lambda) d \lambda .
$$

Using (2.2), we have the existence of a real $C_{1}>0$ such that

$$
|a(k, \xi)| \leqslant C_{1}\left(1+|\xi|^{p(k)-1}\right) \quad \text { for all } \quad k \in \mathbb{Z} \quad \text { and for all } \quad \xi \in \mathbb{R} \text {. }
$$


Therefore,

$$
\begin{aligned}
\int_{0}^{\xi}|a(k, \lambda)| d \lambda & \leq C_{1} \int_{0}^{\xi}\left(1+|\lambda|^{p(k)-1}\right) d \lambda \\
& \leq C_{1}[\lambda]_{0}^{\xi}+C_{1}\left[\frac{\lambda^{p(k)}}{p(k)}\right]_{0}^{\xi} \\
& \leq C_{1}|\xi|+C_{1} \frac{|\xi|^{p(k)}}{p(k)}
\end{aligned}
$$

One deduces that

$$
\begin{aligned}
\sum_{k=1}^{T+1} A(k-1, \Delta u(k-1)) & \leq C_{1} \sum_{k=1}^{T+1}|\Delta u(k-1)|+C_{1} \sum_{k=1}^{T+1} \frac{|\Delta u(k-1)|^{p(k-1)}}{p(k-1)} \\
& \leq C_{1} \sum_{k=1}^{T+1}|\Delta u(k-1)|^{p(k-1)}+\frac{C_{1}}{p^{-}} \sum_{k=1}^{T+1}|\Delta u(k-1)|^{p(k-1)} \\
& \leq(T+1) C_{1}+\left(C_{1}+\frac{C_{1}}{p^{-}}\right) \sum_{k=1}^{T+1}|\Delta u(k-1)|^{p(k-1)}
\end{aligned}
$$

Using the hypothesis (1.3) and according to the above result we have

$$
\begin{aligned}
\widehat{M}\left(\sum_{k=1}^{T+1} A(k-1, \Delta u(k-1))\right) & \leq \frac{B_{2}}{\alpha}\left(\sum_{k=1}^{T+1} A(k-1, \Delta u(k-1))\right)^{\alpha} \\
& \leq \frac{B_{2}}{\alpha}\left[(T+1) C_{1}+\left(C_{1}+\frac{C_{1}}{p^{-}}\right) \sum_{k=1}^{T+1}|\Delta u(k-1)|^{p(k-1)}\right]^{\alpha} \\
& \leq \frac{B_{2}}{\alpha}\left[\left((T+1) C_{1}\right)+\left(\left(C_{1}+\frac{C_{1}}{p^{-}}\right) \sum_{k=1}^{T+1}|\Delta u(k-1)|^{p(k-1)}\right)\right]^{\alpha} .
\end{aligned}
$$

As function $x \longmapsto x^{\alpha}$ being convex for all $\alpha>1$, then we have the inequality

$$
(a+b)^{\alpha} \leq 2^{\alpha-1}\left(a^{\alpha}+b^{\alpha}\right), \text { for all } a, b \in \mathbb{R} .
$$

Consequently, we have

$$
\widehat{M}\left(\sum_{k=1}^{T+1} A(k-1, \Delta u(k-1))\right) \leq \frac{B_{2}}{\alpha} 2^{\alpha-1}\left[\left((T+1) C_{1}\right)^{\alpha}+\left(C_{1}+\frac{C_{1}}{p^{-}}\right)^{\alpha}\left(\sum_{k=1}^{T+1}|\Delta u(k-1)|^{p(k-1)}\right)^{\alpha}\right] .
$$

Using the relation $\left(d_{3}\right)$ we get

$$
\begin{aligned}
\widehat{M}\left(\sum_{k=1}^{T+1} A(k-1, \Delta u(k-1))\right) & \leq \frac{B_{2}}{\alpha} 2^{\alpha-1}\left[(T+1)^{\alpha} C_{1}^{\alpha}+\left(C_{1}+\frac{C_{1}}{p^{-}}\right)^{\alpha}(T+1)^{\alpha}\left(2^{p^{+}}\|u\|_{e}^{p^{+}}+1\right)^{\alpha}\right] \\
& \leq \frac{B_{2}}{\alpha} 2^{\alpha-1}(T+1)^{\alpha}\left[C_{1}^{\alpha}+\left(C_{1}+\frac{C_{1}}{p^{-}}\right)^{\alpha} 2^{\alpha-1}\left(2^{\alpha p^{+}}\|u\|_{e}^{\alpha p^{+}}+1\right)\right] \\
& \leq \frac{B_{2}}{\alpha} 2^{\alpha-1}(T+1)^{\alpha} C_{1}^{\alpha}+\frac{B_{2}}{\alpha}\left(C_{1}+\frac{C_{1}}{p^{-}}\right)^{\alpha}(T+1)^{\alpha} 2^{2 \alpha-2} \\
& +\frac{B_{2}}{\alpha}\left(C_{1}+\frac{C_{1}}{p^{-}}\right)^{\alpha}(T+1)^{\alpha} 2^{2 \alpha-2+\alpha p^{+}}\|u\|_{e}^{\alpha p^{+}} .
\end{aligned}
$$

According to $\left(d_{4}\right)$, we have

$$
F(k, u) \geq \alpha_{1}(k)|u|^{s(k)}+\alpha_{2}(k)
$$




\section{Consequently,}

$$
-\lambda \sum_{k=1}^{T} F(k, u) \leq-\lambda \sum_{k=1}^{T}\left(\alpha_{1}(k)|u(k)|^{s(k)}+\alpha_{2}(k)\right) .
$$

Using $\left(d_{2}\right)$ we obtain

$$
-\lambda \sum_{k=1}^{T} F(k, u) \leq-\lambda \alpha_{1}^{-} T^{\frac{2-s^{-}}{2}}\|u\|_{e}^{s^{-}}-\lambda \alpha_{2}^{-} T .
$$

Since $J$ is continuous, there are positive constants $m_{1}$ and $m_{2}$ such that $J(u) \leq m_{1}|u(0)|+m_{2}|u(T+1)| \leq$ $C_{2}\|u\|_{\infty}$, where $C_{2}=m_{1}+m_{2}$. In addition, by equivalent of norms on $H_{T}$, there is some $C_{3}>0$ such that $C_{2}\|u\|_{\infty} \leq C_{3}\|u\|_{e}^{\alpha p^{+}}$.

Finally, we get

$$
\begin{aligned}
\Gamma_{T}(u) & \leq \frac{B_{2}}{\alpha} 2^{\alpha-1}(T+1)^{\alpha} C_{1}^{\alpha}+\frac{B_{2}}{\alpha}\left(C_{1}+\frac{C_{1}}{p^{-}}\right)^{\alpha}(T+1)^{\alpha} 2^{2 \alpha-2}+\frac{B_{2}}{\alpha}\left(C_{1}+\frac{C_{1}}{p^{-}}\right)^{\alpha}(T+1)^{\alpha} 2^{2 \alpha-2+\alpha p^{+}}\|u\|_{e}^{\alpha p^{+}} \\
& -\lambda \alpha_{1}^{-} T^{\frac{2-s^{-}}{2}}\|u\|_{e}^{s^{-}}-\lambda \alpha_{2}^{-} T+C_{3}\|u\|_{e}^{\alpha p^{+}} .
\end{aligned}
$$

Since $s^{-}>\alpha p^{+}$, we see that $\Gamma_{T}$ is anti-coercive on $H_{T}$.

Lemma 5.2. Assume that conditions (2.1), (2.2), (1.3) and $\left(d_{4}\right)$ hold with $s^{-}=\alpha p^{+}$. Then, the functional $\Gamma_{T}$ is anti-coercive on $H_{T}$, for any $\lambda \in\left(\lambda_{0},+\infty\right)$, where

$$
\lambda_{0}=\frac{T^{\frac{s^{-}-2}{2}}}{\alpha_{1}^{-} \alpha}\left[(2)^{2 \alpha-2+s^{-}}(T+1)^{\alpha} B_{2} C_{1}^{\alpha}\left(\frac{p^{-}+1}{p^{-}}\right)^{\alpha}+C_{3}\right] .
$$

Proof.

According to the proof of Lemma 5.2, we get

$$
\begin{aligned}
\Gamma_{T}(u) & \leq\left[\frac{B_{2}}{\alpha}\left(C_{1}+\frac{C_{1}}{p^{-}}\right)^{\alpha}(T+1)^{\alpha} 2^{2 \alpha-2+s^{-}}-\lambda \alpha_{1}^{-} T^{\frac{2-s^{-}}{2}}+C_{3}\right]\|u\|_{e}^{s^{-}}+\frac{B_{2}}{\alpha} 2^{\alpha-1}(T+1)^{\alpha} C_{1}^{\alpha} \\
& +\frac{B_{2}}{\alpha}\left(C_{1}+\frac{C_{1}}{p^{-}}\right)^{\alpha}(T+1)^{\alpha} 2^{2 \alpha-2}-\lambda \alpha_{2}^{-} T .
\end{aligned}
$$

Thus, $\Gamma_{T}$ is anti-coercive on $H_{T}$ for any $\lambda \in\left(\lambda_{0},+\infty\right)$.

Note that for any $p^{+} \geq 1$, the functional $\|\cdot\|_{p^{+}}: Y_{a} \longrightarrow \mathbb{R}$ defined by

$$
\|u\|_{p^{+}}:=\left(\sum_{k=1}^{T+1}|\Delta u(k-1)|^{p^{+}}\right)^{\frac{1}{p^{+}}}
$$

is a norm on $Y_{a}$, while it is obviously not a norm on $H_{T}$. Since all norms on $Y_{a}$ are equivalent, therefore, there exists a constant $\zeta>0$ such that

$$
\sum_{k=1}^{T+1}|\triangle u(k-1)|^{p^{+}} \geq \zeta\|u\|_{e}^{p^{+}}
$$

for all $u \in Y_{a}$.

Theorem 5.3. Assume that conditions (1.3),(2.1)-(2.6), $\left(a_{1}\right)-\left(a_{3}\right)$ and $\left(d_{4}\right),\left(d_{5}\right),\left(d_{6}\right)$ hold with $s^{-}>\alpha p^{+}$. Let $\lambda>0$ be fixed. Then, problem (1.1) has at least three T-periodic solutions, at least two of which are non-trivial. 
Proof.

Let $\epsilon>0$ a real number satisfying

$$
\epsilon \leq \frac{B_{1} \zeta^{\alpha}}{\alpha \lambda T\left(p^{+}\right)^{\alpha}}
$$

By $\left(d_{5}\right)$ and $\left(d_{6}\right)$ there exists $\rho \in\left(0, \omega_{0}\right)$ with $\omega_{0}<\frac{1}{4}$ such that

$$
F(k, u) \leq \epsilon|u|^{s^{-}} \text {for }|u| \leq \rho .
$$

If $u \in Y_{a}$ with $\|u\| \leq \rho$, then, $|u(k)| \leq \rho$ for all $k \in \mathbb{Z}$.

From assumptions (1.3) and (2.5),

$$
\begin{aligned}
\widehat{M}\left(\sum_{k=1}^{T+1} A(k-1, \Delta u(k-1))\right) & \geq \frac{B_{1}}{\alpha}\left(\sum_{k=1}^{T+1} A(k-1, \Delta u(k-1))\right)^{\alpha} \\
& \geq \frac{B_{1}}{\alpha}\left(\frac{1}{p(k-1)} \sum_{k=1}^{T+1}|\Delta u(k-1)|^{p(k-1)}\right)^{\alpha} \\
& \geq \frac{B_{1}}{\alpha}\left(\frac{1}{p^{+}} \sum_{k=1}^{T+1}|\Delta u(k-1)|^{p^{+}}\right)^{\alpha} .
\end{aligned}
$$

By using (5.1), it follows that

$$
\widehat{M}\left(\sum_{k=1}^{T+1} A(k-1, \Delta u(k-1))\right) \geq \frac{B_{1}}{\alpha\left(p^{+}\right)^{\alpha}} \zeta^{\alpha}\|u\|_{e}^{\alpha p^{+}} .
$$

By using (5.2), we have

$$
-\lambda \sum_{k=1}^{T} F(k, u) \geq-\lambda \epsilon \sum_{k=1}^{T}|u(k)|^{s^{-}} .
$$

Consequently,

$$
\begin{aligned}
\Gamma_{T}(u) & \geq \frac{B_{1}}{\alpha\left(p^{+}\right)^{\alpha}} \zeta^{\alpha}\|u\|_{e}^{\alpha p^{+}}-\lambda \epsilon \sum_{k=1}^{T}|u(k)|^{s^{-}}-C_{3}\|u\|_{e}^{\alpha p^{+}} \\
& \geq \frac{B_{1}}{\alpha\left(p^{+}\right)^{\alpha}} \zeta^{\alpha}\|u\|_{e}^{\alpha p^{+}}-\lambda \epsilon T\|u\|_{e}^{s^{-}}-C_{3}\|u\|_{e}^{\alpha p^{+}} \\
& \geq \frac{B_{1}}{\alpha\left(p^{+}\right)^{\alpha}} \zeta^{\alpha}\|u\|_{e}^{s^{-}}-\lambda \epsilon T\|u\|_{e}^{s^{-}}-C_{3}\|u\|_{e}^{\alpha p^{+}} \\
& \geq\|u\|_{e}^{s^{-}}\left(\frac{B_{1}}{\alpha\left(p^{+}\right)^{\alpha}} \zeta^{\alpha}-\lambda \epsilon T-C_{3}\right) \\
& \geq 0 .
\end{aligned}
$$

Thus, the above relation together with $(2.1)$ and $\left(a_{3}\right)$ yields

$$
\Gamma_{T}(u) \geq \Gamma_{T}(0) \text { for all } u \in Y_{a} \text { with }\|u\|_{e} \leq \rho .
$$

Note also that for every $u \in W_{a}$, we have $\Delta u(k-1)=0$, for all $k \in \mathbb{Z}$ and then, according to $(2.1), A(k, 0)=0$; so,

$$
\Gamma_{T}(u)=-\lambda \sum_{k=1}^{T} F(k, u(k))+J(u),
$$


for all $u \in W_{a}, \forall a \in \mathbb{R}$. If $u \in W_{a}$ with $\|u\|_{e} \leq \rho$, then $|u(k)| \leq \omega_{0}$ for all $k \in \mathbb{Z}$. Thus, by $\left(d_{4}\right)$ and $\left(a_{3}\right)$ it follows that

$$
\Gamma_{T}(u) \leq \Gamma_{T}(0) \text { for all } u \in W_{a} \text {, with }\|u\|_{e} \leq \rho \text { where } a \in \mathbb{R} \text {. }
$$

Let $\Psi_{T}=-\Gamma_{T}(u)$. Using Lemma 5.2, we deduce that $\Psi_{T}$ satisfies the (PS) condition. Note that $\Psi_{T}$ is bounded from below; moreover, as $\Psi_{T}$ is coercive and continuous, it admits a minimizer. Using $\left(b_{2}\right)$ we obtain

$$
\inf _{u \in H_{T}} \Psi(u)=-\sup _{u \in H_{T}} \Gamma(u)<0 .
$$

We have shown that assumptions of Theorem 2.2 are satisfied, so $\Psi_{T}$ has at least three critical points, at least two of them are non-zero critical points. By Lemma 4.3, these are non-trivial $T$-periodic solutions of problem (1.1).

In the case $s^{-}=\alpha p^{+}$we have the following special case.

Corollary 5.4. Assume that conditions $(2.1)-(2.6),\left(a_{1}\right)-\left(a_{3}\right)$ and $\left(d_{4}\right),\left(d_{5}\right),\left(d_{6}\right)$ hold with $s^{-}=p^{+}$. Let $\lambda \in\left(\lambda_{0},+\infty\right)$. Then, problem (1.1) has at least three T-periodic solutions, at least two of which are non-trivial.

\section{Multiple SOlutions OF PROBLEM (1.1) BY THREe CRITICAL POINTS THEOREM}

Now we provide multiplicity results for the problem (1.1) using Theorem 3.3.

Let functionals $\phi, \mu: H_{T} \longrightarrow \mathbb{R}$ be defined by

$$
\varphi(u):=\widehat{M}\left(\sum_{k=1}^{T+1} A(k-1, \Delta u(k-1))\right) \text { and } \mu(u):=-\sum_{k=1}^{T} F(k, u(k)) .
$$

Then, we see that $\Gamma_{T}=\varphi+\lambda \mu+J$.

Assume that $F$ has the following properties.

$\left(A_{1}\right):$ There exists a constant $C \in \mathbb{R}$ such that

$$
F(k, u) \leq C \text { for all }(k, u) \in \mathbb{Z} \times \mathbb{R}
$$

$\left(A_{2}\right):$ There exist numbers $\tau_{1}, \tau_{2}, \tau_{3}>0, \tau_{3}>\tau_{2}>\tau_{1}$ such that for all $k \in \mathbb{Z}:$

$\left(A_{2}^{\prime}\right) F(k, u)<0$ with $0<|u| \leq \tau_{1}$,

$\left(A_{2}^{\prime \prime}\right) F(k, u)>0$ with $\tau_{2} \leq|u| \leq \tau_{3}$.

Example 6.1. As example of function satisfying assumptions $\left(a_{1}\right)-\left(a_{3}\right)$ and $\left(A_{1}\right)-\left(A_{2}\right)$, we can give the following, where $T \geq 2$ is a fixed even natural number.

$F: \mathbb{Z} \times \mathbb{R} \longrightarrow \mathbb{R}$ given by

$$
F(k, t):=\left\{\begin{array}{l}
-\sin |t| \text { if }|t| \leq \pi, \\
|t-\pi| \text { if } \pi<|t|<2 \pi, \\
\pi \text { if }|t| \geq 2 \pi .
\end{array}\right.
$$

In this paragraph we will consider the problem (1.1) on $Y_{a}$. 
Remark 6.1. The functional $\varphi$ is not coercive on $H_{T}$, but it is coercive on $Y_{a}$, for $a \in \mathbb{R}$. Indeed, given a sequence $\left(U_{n}\right)_{n \in \mathbb{N}}$ such that $U_{n}(k)=$ a for all $k \in \mathbb{Z}$, we see that

$$
\varphi\left(u_{n}\right)=\widehat{M}\left(\sum_{k=1}^{m} A\left(k-1, \Delta u_{n}(k-1)\right)\right)=\widehat{M}\left(\sum_{k=1}^{m} A(k-1,0)\right)=0 .
$$

Letting $a \longrightarrow+\infty$, we see that $\varphi$ is not coercive on $H_{T}$. Furthermore, by using (5.3) we get

$$
\widehat{M}\left(\sum_{k=1}^{T+1} A(k-1, \Delta u(k-1))\right) \geq \frac{B_{1}}{\alpha\left(p^{+}\right)^{\alpha}} \zeta^{\alpha}\|u\|_{e}^{\alpha p^{+}} .
$$

Therefore, $\varphi$ is coercive on $Y_{a}$.

Note that since we obtain solutions in $Y_{a}$, we know that there are not constant solutions, however there may still be a zero solution.

Theorem 6.1. Assume that conditions $(1.3),(2.1)-(2.6),\left(a_{1}\right)-\left(a_{3}\right),\left(A_{1}\right)$ and $\left(A_{2}\right)$ hold. Then, there exists $\lambda>0$ such that problem (1.1) has at least three solutions in $Y_{a}$, at least two of which are necessarily non-zero.

Proof.

According to the previous results, $\varphi$ is coercive on $Y_{a}$ and is $C^{1}$, therefore, $\varphi$ checks assumptions of Theorem 3.3.

According to the hypothesis $\left(A_{1}\right)$,

$$
F(k, u(k)) \leq C \Longrightarrow \Gamma(u) \geq-T C>-\infty .
$$

Hence, $\Gamma$ is bounded from below.

By $\left(A_{2}^{\prime \prime}\right)$ it follows that

$$
-F(k, u(k))<0,
$$

for all $u \in Y_{a}$ such that $\tau_{2} \leq|u(k)| \leq \tau_{3}$ for all $k \in \mathbb{Z}$. We thus deduce that there exists a point $u \in Y_{a}$ such that $\mu(u)<0$. Consequently,

$$
\inf _{u \in Y_{a}} \mu(u)<0 .
$$

By $\left(A_{2}^{\prime}\right)$ and since $\varphi$ is continuous, coercive, convex, non-negative and $\varphi(0)=0$, we get that there are $s, r>0$, such that $\mu(u)>0$ for $r \leq \varphi(u) \leq s$. Therefore, $\left(b_{2}\right)$ is satisfied.

Now, by $\left(a_{3}\right)$ and $\left(A_{2}^{\prime \prime}\right)$, we obtain

$$
\mu(0)=0<\inf _{r \leq \varphi(u) \leq s} \mu(u) .
$$

Hence, condition $\left(b_{3}\right)$ is satisfied. Thus, by Theorem 3.3 we see that there exists $\lambda>0$ such that the functional $\Gamma_{T}$ has at least three critical points on $Y_{a}$. Since by Lemma 4.3, critical points of $\Gamma_{T}$ are solutions of problem (1.1), hence the result follows.

For numbers $r_{0}, s_{0}>0$, we put

$$
r^{\prime}:=\inf \left\{\|u\|_{\max }: \varphi(u) \geq r_{0}\right\} \text { and } s^{\prime}:=\sup \left\{\|u\|_{\max }: \varphi(u) \leq s_{0}\right\},
$$


where

$$
\|u\|_{\max }=\max _{k \in \mathbb{Z}[1, T]}|u(k)|
$$

Hence,

$$
\left\{u \in Y_{a}: \varphi(u) \geq r_{0}\right\} \subset\left\{u \in Y_{a}:\|u\|_{\max } \geq r^{\prime}\right\}
$$

and

$$
\left\{u \in Y_{a}: \varphi(u) \leq s_{0}\right\} \subset\left\{u \in Y_{a}:\|u\|_{\max } \leq s^{\prime}\right\} .
$$

Assume that $F$ satisfies the following.

$\left(A_{3}\right)$ : There exist constants $r_{0}, s_{0}>0, r_{0}<s_{0}$ such that the following relations hold.

$\left(A_{3}^{\prime}\right) \sup _{|u|<s^{\prime}} F\left(k_{0}, u\right)<\sup _{u \in \mathbb{R}} F(k, u)$ for some $k_{0} \in \mathbb{Z}[1, T] ;$

$\left(A_{3}^{\prime \prime}\right) F(k, u) \leq 0$ for all $(k, u) \in \mathbb{Z}[1, T] \times \mathbb{R}$ with $r^{\prime} \leq|u| \leq s^{\prime}$.

Remark 6.2. Note that $\left(A_{3}^{\prime}\right)$ is equivalent to the following assumptions.

$$
\sum_{k=1}^{T} \sup _{|t| \leq s^{\prime}} F(k, t)<\sum_{k=1}^{T} \sup _{t \in \mathbb{R}} F(k, t) ;
$$

In fact, from (6.1) it is easy to get $\left(A_{2}^{\prime}\right)$, since

$$
\sup _{|t| \leq s^{\prime}} F(k, t) \leq \sup _{t \in \mathbb{R}} F(k, t) \text { for any } s^{\prime}>0 \text { and for any } k \in \mathbb{Z}[1, T] .
$$

On the other hand, (6.1) is equivalent to the following.

$$
\left(\sup _{t \in \mathbb{R}} F(1, t)-\sup _{|t|<s^{\prime}} F(1, t)\right)+\left(\sup _{t \in \mathbb{R}} F(2, t)-\sup _{|t|<s^{\prime}} F(2, t)\right)+\ldots+\left(\sup _{t \in \mathbb{R}} F(T, t)-\sup _{|t|<s^{\prime}} F(T, t)\right)>0, \text { we }
$$
get (6.1), noting again (6.2). We assume that (6.1) was used in the context of anisotropic problems in [10].

Theorem 6.2. Let $r_{0}, s_{0}>0, r_{0}<s_{0}$. Assume that conditions (1.3), (2.1) - (2.6), $\left(a_{1}\right)-\left(a_{3}\right),\left(A_{1}\right)$ and $\left(A_{3}\right)$ hold. Then, there exists $\lambda>0$ such that problem (1.1) has at least three solutions on $Y_{a}$, at least two of which are non-trivial.

Proof.

We will show that $\Gamma_{T}$ verifies assumptions of Theorem 3.3. Clearly, $\varphi$ is coercive and is of class $C^{1}$ on $Y_{a}, \varphi(0)=0$ and $\mu$ is bounded from below. By $\left(A_{3}^{\prime}\right)$, we deduce that

$$
\begin{aligned}
\inf _{u \in Y_{a}} \mu(u) & =-\sum_{k=1}^{T} \sup _{\{i \in \mathbb{Z}[1, T]: u(i) \in \mathbb{R}\}} F(k, u(i)) \\
& <-\sum_{k=1}^{T} \sup _{\left\{i \in \mathbb{Z}[1, T]: u(i) \leq s^{\prime}\right\}} F(k, u(i))=\inf _{\|u\|_{\text {max }} \leq s^{\prime}} \mu(u) \leq \inf _{\varphi(u) \leq s_{0}} \mu(u) .
\end{aligned}
$$


Thus, $\left(b_{2}\right)$ is satisfied. For any $u \in Y_{a}$ with $r_{0} \leq \varphi(u) \leq s_{0}$, we have $r^{\prime} \leq\|u\|_{\text {max }} \leq s^{\prime}$.

By $\left(A_{3}^{\prime \prime}\right)$, we obtain

$$
\begin{aligned}
\inf _{r_{0} \leq \varphi(u) \leq s_{0}} \mu(u) & \geq \inf _{r^{\prime} \leq\|u\|_{\text {max }} \leq s^{\prime}} \mu(u) \\
& =-\sum_{k=1}^{T}\left\{\sup _{\left\{i \in \mathbb{Z}[1, T]: r^{\prime} \leq \max _{i \in \mathbb{Z}[1, T]}|u(i)| \leq s^{\prime}\right\}} F(k, u(i)) \geq 0=\mu(0) .\right.
\end{aligned}
$$

Hence, $\left(b_{3}\right)$ is satisfied. Finally, by Theorem 3.3, there exists $\lambda>0$ such that the functional $\Gamma_{T}$ has at least three critical points in $Y_{a}$. Consequently, by Lemma 4.3, problem (1.1) has at least three solutions.

We note that a type of small solutions can be obtained by lying near the origin, without assuming $\left(A_{3}^{\prime}\right)$.

Let us consider the following remark.

Remark 6.3. Let $k_{0} \in \mathbb{Z}[1, T]$ be a fixed real. Since $F\left(k_{0}, t\right) \leq C$ for some $C>0$, so

$$
\sup _{t \in \mathbb{R}} F\left(k_{0}, t\right)=\beta \leq C .
$$

If $\beta>0$ we can reason as follows. Since $t \longmapsto F\left(k_{0}, t\right)$ is continuous and since $F\left(k_{0}, 0\right)=0$, there exists a sufficiently small positive constant s' depending on $\beta$ such that

$$
-\frac{\beta}{2} \leq F\left(k_{0}, t\right) \leq \frac{\beta}{2} .
$$

Thus,

$$
\sup _{|t| \leq s^{\prime}} F\left(k_{0}, t\right)<\sup _{t \in \mathbb{R}} F\left(k_{0}, t\right) .
$$

From the above remark, we have the following result.

Corollary 6.3. Assume that conditions $(1.3),(2.1)-(2.6),\left(a_{1}\right)-\left(a_{3}\right),\left(A_{1}\right)$ and $\left(A_{3}\right)$ hold. Let

$$
\sup _{t \in \mathbb{R}} F\left(k_{0}, t\right)>0,
$$

for some $k_{0} \in \mathbb{Z}[1, T]$. Then, there exists $\lambda>0$ such that problem (1.1) has at least three solutions on $Y_{a}$, for a $\in \mathbb{R}$, at least two of which are non-trivial.

We can also replace $\left(A_{3}^{\prime \prime}\right)$ with $\left(A_{2}^{\prime}\right)$ provided that $\tau_{1}$ is sufficiently large. Indeed, we can reason as follows.

Remark 6.4. Let $s_{0}>\frac{B_{1} \zeta^{\alpha}}{\alpha\left(p^{+}\right)^{\alpha}}, 0<r_{0}<s_{0}$ and $\tau_{0}=\zeta_{0}\left[\frac{s_{0} \alpha\left(p^{+}\right)^{\alpha}}{B_{1} \zeta^{\alpha}}\right]^{\frac{1}{\alpha p^{+}}}$. If $\left(A_{2}^{\prime}\right)$ is satisfied with $\tau_{1} \geq \tau_{0}$, then $\left(A_{3}^{\prime \prime}\right)$ is also satisfied with $s^{\prime}=\tau_{0}$. The inequality $\varphi(u) \leq s_{0}$ is equivalent to

$$
\widehat{M}\left(\sum_{k=1}^{T+1} A(k-1, \Delta u(k-1))\right) \leq s_{0}
$$

From (5.3), one has $\frac{B_{1}}{\alpha\left(p^{+}\right)^{\alpha}} \zeta^{\alpha}\|u\|_{e}^{\alpha p^{+}} \leq s_{0}$. Therefore, since $s_{0}>\frac{B_{1} \zeta^{\alpha}}{\alpha\left(p^{+}\right)^{\alpha}}$, we get

$$
\|u\|_{e} \leq\left[\frac{s_{0} \alpha\left(p^{+}\right)^{\alpha}}{B_{1} \zeta^{\alpha}}\right]^{\frac{1}{\alpha p^{+}}}
$$


Since all norms on $Y_{a}$, for $a \in \mathbb{R}$ are equivalent, then, there exists a constant $\zeta_{0}>0$ such that $\|u\|_{\text {max }} \leq \zeta_{0}\|u\|_{e} ;$ hence,

$$
\|u\|_{\max } \leq \zeta_{0}\left[\frac{s_{0} \alpha\left(p^{+}\right)^{\alpha}}{B_{1} \zeta^{\alpha}}\right]^{\frac{1}{\alpha p^{+}}}
$$

and

$$
s^{\prime}=\sup \left\{\|u\|_{\max }: \mu(u) \leq s_{0}\right\}=\zeta_{0}\left[\frac{s_{0} \alpha\left(p^{+}\right)^{\alpha}}{B_{1} \zeta^{\alpha}}\right]^{\frac{1}{\alpha p^{+}}} .
$$

Furthermore,

$$
\left\{u \in Y_{a}: \varphi(u) \leq s_{0}\right\} \subset\left\{u \in Y_{a}:|u| \leq \zeta_{0}\left[\frac{s_{0} \alpha\left(p^{+}\right)^{\alpha}}{B_{1} \zeta^{\alpha}}\right]^{\frac{1}{\alpha p^{+}}}\right\} \subset\left\{u \in Y_{a}:|u| \leq \tau_{1}\right\}
$$

Corollary 6.4. Let $s_{0}>\frac{B_{1} \zeta^{\alpha}}{\alpha\left(p^{+}\right)^{\alpha}}, 0<r_{0}<s_{0}$ and $\tau_{0}=\zeta_{0}\left[\frac{s_{0} \alpha\left(p^{+}\right)^{\alpha}}{B_{1} \zeta^{\alpha}}\right]^{\frac{1}{\alpha p^{+}}}$. Assume that conditions (1.3), (2.1) (2.6), $\left(a_{1}\right)-\left(a_{3}\right),\left(A_{1}\right)$ and $\left(A_{3}\right)$ hold. If moreover $\left(A_{2}^{\prime}\right)$ is satisfied with $\tau_{1} \geq \tau_{0}$, then, there exists $\lambda>0$ such that problem (1.1) has at least three solutions on $Y_{a}$, for $a \in \mathbb{R}$, at least two of which are non-trivial.

Analogously, we obtain the following.

Corollary 6.5. Let $s_{0} \leq \frac{B_{1} \zeta^{\alpha}}{\alpha\left(p^{+}\right)^{\alpha}}, \quad 0<r_{0}<s_{0}$ and $\tau_{0}=\zeta_{0}\left[\frac{s_{0} \alpha\left(p^{+}\right)^{\alpha}}{B_{1} \zeta^{\alpha}}\right]^{\frac{1}{\alpha p^{-}}}$. Assume that conditions (1.3), (2.1)(2.6), $\left(a_{1}\right)-\left(a_{3}\right),\left(A_{1}\right)$ and $\left(A_{3}\right)$ hold. If moreover $\left(A_{2}^{\prime}\right)$ is satisfied with $\tau_{1} \geq \tau_{0}$, then there exists $\lambda>0$ such that problem (1.1) has at least three solutions on $Y_{a}$, for $a \in \mathbb{R}$, at least two of which are non-trivial.

\section{REFERENCES}

[1] R.P. Agarwal, K. Perera, D. O'Regan Multiple positive solutions of singular and nonsingular discrete problems via variational methods, Nonlinear Anal. 58 (2004), 69-73.

[2] R.P. Agarwal, K. Perera, D. O'Regan; Multiple positive solutions of singular discrete p-Laplacian problems via variational methods, Adv. Diff. Equ. 2 (2005), 93-99.

[3] G. Bonanno, P. Candito, Nonlinear difference equations investigated via critical point methods, Nonlinear Anal. 70 (2009), $3180-3186$.

[4] A. Cabada, A. Lannizzotto, S. Tersian, Multiple solutions for discrete boundary value problems, J. Math. Anal. Appl. 356 (2009), 418-428.

[5] X. Cai, J. Yu, Existence theorems for second-order discrete boundary value problems, J. Math. Anal. Appl. 320 (2006), 649-661.

[6] P. Candito, N. Giovannelli, Multiple solutions for a discrete boundary value problem involving the p-Laplacian, Math. Appl. Comput. 56 (2008), 959- 964

[7] K.-C. F. Chang, Variational methods for non-differentiable functionals and their applications to partial differential equations, J. Math. Anal. Appl. 80(1) (1981), 102-129.

[8] F.H. Clarke, Optimization and Nonsmooth Analysis, Classics in Applied Mathematics, Vol. 5, SIAM, Philadelphia, 1990.

[9] M. Galewski, R. Wieteska, Multiple solutions for periodic problems with the discrete $p(k)$-Laplacian, Discrete Contin. Dyn. Syst. Ser. B 19(8) (2014), 2535-2547.

[10] M. Galewski and S. Glab, On the discrete boundary value problem for anisotropic equation, Math. Anal. Appl. 386(2) (2012), 956-965.

[11] A. Guiro, I. Nyanquini, S. Ouaro, On the solvability of discrete nonlinear Neumann problems involving the $p(x)$-Laplacian, Adv. Diff. Equ. 2011 (2011), 32.

[12] P. Jebelean, C. S,erban, Ground state periodic solutions for difference equations with discrete $p$-Laplacian, Appl. Math. Comput. 217 (2011), 9820- 9827.

[13] B. Kone, S. Ouaro, Weak solutions for anisotropic discrete boundary value problems, J. Differ. Equ. Appl. 17(2011), 1537-1547. 
[14] A. Kristaly, V. Radulescu and Cs. Varga, Variational Principles in Mathematical Physics, Geometry, and Economics: Qualitative Analysis of Nonlinear Equations and Unilateral Problems, Encyclopedia of Mathematics and Its Applications, No. 136, Cambridge University Press, Cambridge, 2010.

[15] N. Marcu, G.M. Bisci, Existence and multiplicity of solutions for nonlinear discrete inclusions, Electron. J. Differ. Equ. $2012(2012)$, 192.

[16] R.A. Mashiyev, Z. Yucedag, S. Ogras, Existence and multiplicity of solutions for a Dirichlet problem involving the discrete $p(x)$-Laplacian operator, Electron. J. Qual. Theory Differ. Equ. 2011(2011), 67.

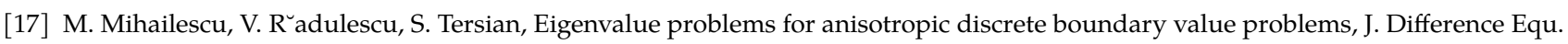
Appl. 15 (2009), 557-567.

[18] D. Motreanu, V. Radulescu, Variational and Non-Variational Methods in Nonlinear Analysis and Boundary Value Problems, Nonconvex Optimization and Its Applications,Vol. 67, Kluwer Academic Publishers, Dordrecht, 2003.

[19] D. Motreanu, Cs Varga, Some critical point result for locally Lipschitz functionals, Commun. Appl. Nonlinear Anal. 4 (1997), 17-33.

[20] S. Ouaro, M. Zoungrana, Multiplicity of solutions to discrete inclusions with the $p(k)$-Laplace Kirchhoff type equations, Asia Pac. J. Math. 5 (1) (2018), 27-49.

[21] X. Wu, A new critical point theorem for locally Lipschitz functionals with applications to differential equations, Nonlinear Anal. 66(3) (2007), 624-638.

[22] J. Yu, Z. Guo, On boundary value problems for a discrete generalized Emden-Fowler equation, J. Math. Anal. Appl. 231 (2006), 18-31.

[23] G. Zhang, S. Liu, On a class of semipositone discrete boundary value problem, J. Math. Anal. Appl. 325 (2007), 175-182. 